\title{
Chinese Herbal Medicine Paratherapy for Parkinson's Disease: A Meta-Analysis of 19 Randomized Controlled Trials
}

\author{
Yan Wang, Cheng-Long Xie, Lin Lu, Deng-Lei Fu, and Guo-Qing Zheng \\ The Center of Neurology and Rehabilitation, The Second Affiliated Hospital of Wenzhou Medical College, Wenzhou 325027, China
}

Correspondence should be addressed to Guo-Qing Zheng, gq_zheng@sohu.com

Received 10 February 2012; Revised 26 April 2012; Accepted 7 May 2012

Academic Editor: Seung-Heon Hong

Copyright () 2012 Yan Wang et al. This is an open access article distributed under the Creative Commons Attribution License, which permits unrestricted use, distribution, and reproduction in any medium, provided the original work is properly cited.

Parkinson's disease (PD) is a common and debilitating neurodegenerative disorder that needs long-term levodopa administration and can result in progressive deterioration of body functions, daily activities and participation. The objective of this meta-analysis evaluates the clinical efficacy and safety of Chinese herbal medicine (CHM) as an adjunct therapy for PD patients. Methodological issues include a systematic literature search between 1950 and April 2011 to identify randomized trials involving CHM adjuvant therapy versus western conventional treatment. The outcome measures assessed were the reduction in scores of Unified Parkinson's Disease Rating Scale (UPDRS) and adverse effects. 19 trials involving 1371 participants were included in the meta-analysis. As compared to western conventional treatment, CHM adjuvant therapy resulted in greater improvement in UPDRS I, II, III, IV scores, and UPDRS I-IV total scores $(P<0.001)$. Adverse effects were reported in 9 studies. The side effects in CHM adjuvant therapy group were generally less than or lighter than the conventional treatment group. In conclusion, CHM adjuvant therapy may potentially alleviate symptoms of PD and generally appeared to be safe and well tolerated by PD patients. However, welldesigned, randomized, placebo-controlled clinical trials are still needed due to the generally low methodological quality of the included studies.

\section{Introduction}

Parkinson's disease (PD) is a common, chronic, and progressive neurodegenerative disorder resulting from the death of the dopamine containing cells in substantia nigra and can cause significant disability and decreased quality of life [1]. However, no treatment till now has been shown to be neuroprotective in $\mathrm{PD}$, which can slow down or even halt the progression of the disease [2]. Owing to the absence of disease-modifying therapies, dopamine replacement therapy is still the most effective symptomatic treatment of $\mathrm{PD}$, but this mainstay of pharmacological treatment is eventually complicated by highly disabling fluctuations and dyskinesias [3]. The PD patients continue to experience progressive deterioration of body functions, daily activities, and participation. Thus, near two-thirds of PD patients worldwide resort to various kinds of complementary or alternative medicine, which may possibly influence the motor and/or nonmotor symptoms of PD, and/or the effectiveness of dopaminergic therapy, to alleviate the progressive functional disabilities caused by the disease [4].

In Mainland China, the prevalence of PD for those aged 65 years or older was $1.7 \%$, which suggested a similar prevalence with the developed countries [5]. However, China faces the largest number of patients with PD because it has one-fifth of the world's population (1.34 billion in 2011). Therefore, the burden of PD prevention and treatment in China is much higher than that in the developed countries. Fortunately, there is one important characteristic of China's national medical system, that is, traditional Chinese medicine (TCM) and western medicine complement and cooperate with each other, being responsible for the health care of Chinese people together [6]. TCM has played an important role in the medical care of PD patients for thousands of years in China [7]. In modern time, TCM therapy is still widely used for PD treatment, and the application covers about three-fourths of the areas in China [6]. In the past decades, several compressive and systematic 
reviews have focused on TCM for PD treatment [8-10]. However, there is still a lack of reliable scientific evidences for the application of TCM therapy on PD. Recently, some high-quality trials have been published in China [6], and it is timely to reevaluate the existence of evidences. The objective of this meta-analysis therefore is to assess clinical efficacy and safety of Chinese herbal medicine (CHM) as an adjunct therapy of patients suffering from PD.

\section{Methods}

This meta-analysis is conducted according to the preferred reporting items for systematic reviews and meta-analysis: The PRISMA Statement [11].

2.1. Eligibility Criteria. Participants were of any age or sex with idiopathic PD diagnosed according to the UK Brain Bank criteria [12] or Chinese National Diagnosis Standard (CNDS) for PD in 1984 [13] or CNDS updated version in 2006 for PD [14]. The CNDS for PD in 1984 [13] is mainly based on clinical observations: (1) to have at least two of the four typical symptoms and signs (bradykinesia, rest tremor, rigidity, and postural reflex disturbance); (2) whether there is atypical symptoms or signs that does not support the diagnosis of idiopathic Parkinson's disease, such as pyramidal signs, apraxia of gait disorders, cerebellar symptoms, intentional tremor, gaze palsy, severe autonomic dysfunction, obvious dementia associated with mild extrapyramidal symptoms; (3) decrease of homovanillic acid in cerebrospinal fluid is helpful for the definite diagnosis of early Parkinson's disease, and for the differential diagnosis of idiopathic tremor, druginduced parkinsonism, and Parkinson's disease. The CNDS updated version in 2006 for PD [14] was definitions of comparable with the UK Brain Bank criteria [12].

Interventions were any form of CHMs in any dose as adjunct therapy for PD. The patients at the trial groups were given CHM therapy in addition to western conventional medication (WCM).

The outcome measures included the evaluation with Unified Parkinson's Disease Rating Scale (UPDRS) [15], and the adverse events at the end of the treatment course lasting for at least 12 weeks ( 3 months). The UPDRS has long been used as the major rating scale that is used for assessing severity of symptoms of PD. The UPDRS scale consists of the following four segments: Part I (mentation, behavior, and mood) addresses mental dysfunction and mood; Part II (activities of daily living, ADL) assesses motor disability; Part III (motor section) evaluates motor impairment; Part IV (complications) assesses treatment related motor and nonmotor complications.

Only randomized controlled trials (RCTs) were included in the study, regardless of blinding, publication status or language. Quasi-RCTs were not considered such as using the admission sequence for treatment allocation.

2.2. Search Strategy. We electronically searched CENTRAL (The Cochrane Library 2011, Issue 1), PubMed (1950-April 2011), EMBASE (1980-2010), China Hospital Knowledge Database (CHKD, 1979-April 2011), and Wanfang Med
Online Database (WMOD, 1998-April 2011). A list of Chinese and English journals that had the potential to include eligible studies was hand-searched. A manual search of conference proceedings relevant to this topic, references from relevant reports of clinical trials or review articles was performed to retrieve all potentially relevant published and unreported material.

The following search strategy was used: the crossreferenced TCM/CHM and its proprietary names with PD and its derivations, all as $\mathrm{MeSH}$ and as free-text words. The Medical Subject Headings (MeSHs) and text keywords $\mathrm{TCM}$ /all subheadings, CHM/all subheadings in combination with Parkinson's, Parkinson's disease, and PD were utilized.

2.3. Study Selection and Data Extraction. Two review authors (WY, XCL) independently scanned the titles and abstracts to select potential references. Full articles for all potentially relevant trials were retrieved. The two review authors then independently read the selected papers and made a final selection decision. All disagreements were resolved by discussion or by involving a third party author (ZGQ).

A standardized data extraction form was used to extract data, including patients, methods, interventions, and outcomes. The reasons for the exclusion of studies were recorded accordingly. For eligible studies, two review authors (WY, $\mathrm{XCL}$ ) extracted the data independently. Disagreements were resolved through consultation with a third party author (ZGQ).

2.4. Risk of Bias in Individual Studies. Two review authors (WY, XCL) independently assessed the risk of bias of included studies, using the twelve criteria recommended by the Cochrane Back Review Group [16]. The items were scored with "yes (+)," "no (-)," or "unsure (?)." Studies were categorized as having a "low risk of bias" when at least six of the 12 criteria were met. We resolved any disagreement through discussion or consultation with a third party author (ZGQ).

2.5. Data Synthesis and Analysis. We analyzed the data using Review Manager (version 5.0). A fixed-effects model or random-effects model was used to investigate the effect of $\mathrm{CHMs}$ on PD across the trials, and weighted mean difference was calculated. Heterogeneity between trial results was tested using a standard chi-square test and we also calculated the $I^{2}$ statistic. Funnel plot analysis is used to detect Publication Bias. The two-tailed $P$ values less than 0.05 were considered statistically significant.

\section{Results}

3.1. Description of Studies. We identified 1223 potentially relevant articles. After screening titles and abstracts, 1156 were excluded because they were studies with nonclinical trials, case reports, lack of comparison group, or efficacy of CHM not being the objective of study. We conducted full-text evaluation on the remaining 67 articles, and 48 more articles were excluded for not meeting our inclusion 


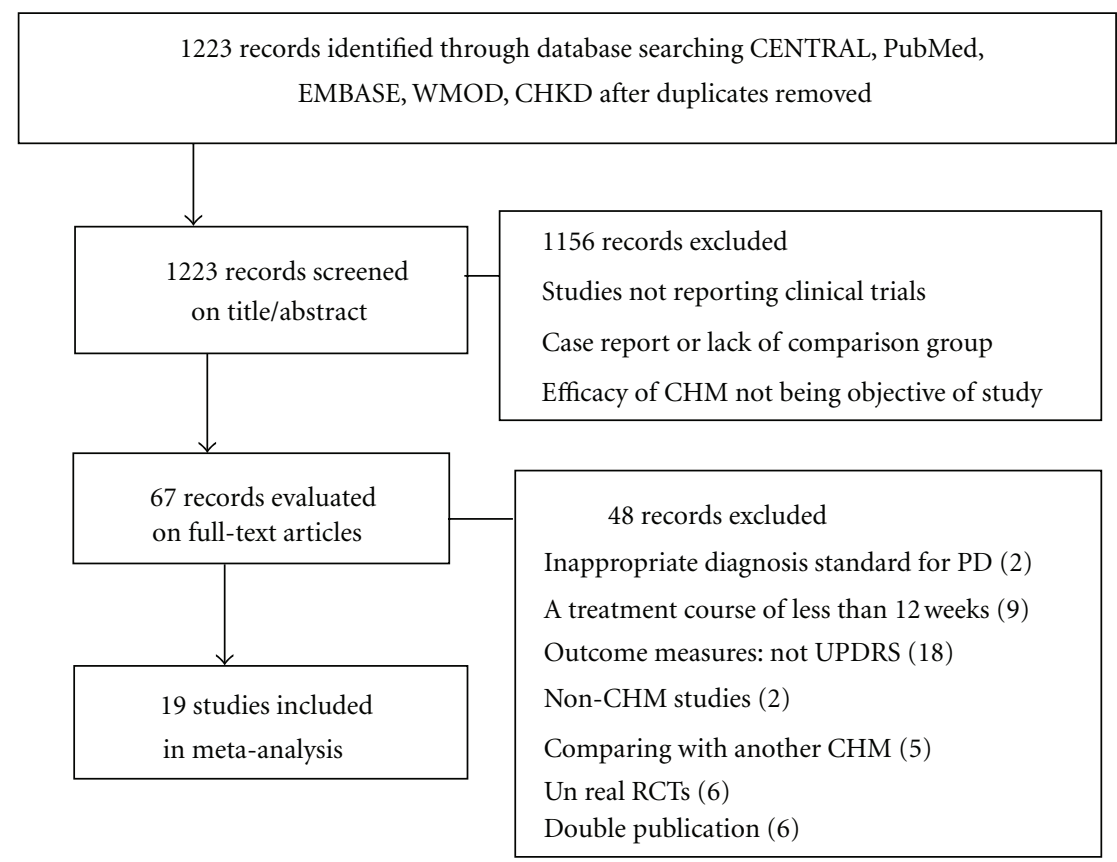

FIGURE 1: Flow diagram for the process of identifying eligible randomized controlled trials. WMOD: Wanfang Med Online Database; CHKD: China Hospital Knowledge Database; CHM: Chinese herbal medicine; PD: Parkinson's disease; UPDRS: the Unified Parkinson's Disease Rating Scale; RCT: randomized controlled trial.

criteria: 2 articles used expert-made diagnosis standard for PD; 9 articles reported a treatment course of less than 12 weeks; 18 articles used homemade rating systems or the Webster rating scale, not UPDRS; 2 articles did not study CHM; 5 articles evaluated CHMs paratherapy by comparing combination treatment of CHM and WCM, or another CHM; 6 studies were not real RCTs; 6 studies were suspected of being published more than once by the authors or publishers. Finally, 19 trials were included in this analysis [17-35]. The screening process is summarized in a flow diagram (Figure 1).

3.2. Characteristics of Included Studies. A total of 1371 participants were included in the 19 studies. All of the trials were conducted in China. 2 articles published in English [27, 35] and 17 articles in Chinese from 2003 to 2011. 16 studies were single-center trials, while the remaining 3 were multicenter trials $[26,28,29]$. There were 825 male and 546 female participants ranging from 35 to 81 years old. 12 studies applied the CNDS (1984 version) for PD; the other 7 studies used CNDS (updated version in 2006) [30, 31, 34] or UK Brain Bank diagnostic criteria [27, 28, 33, 35] for PD. The disease duration ranged from 6 months to 21 years. Except 7 trials $[18,19,23,24,30-32]$, the Hoehn \& Yahr (H \& Y) stage was conducted in 12 trials. All oral CHMs interventions as add-on therapy were investigated by comparing with WCM controls. 4 trials have WCM plus placebo control [26-28, 35]. The course of treatment in all included trials lasted at least 12 weeks ( 3 months). The details of the characteristics of included studies are listed in Table 1.
3.3. Risk of Bias in Included Studies. The twelve criteria recommended by the Cochrane Back Review Group were used to assess the risk of bias [16]. The number of criteria met varied from $2 / 12$ to $11 / 12$ (see Table 2 ). All the included studies indicated randomization, but only 8 trials reported the method of generating random sequences $[17,18,26-29$, $34,35]$, and 5 trials described allocation concealment [18, 26, $28,29,35]$. 5 trials mentioned blinding procedures to both patients and investigators $[26-29,35]$, but only one trial was assessor-blind [26]. 3 trials described intention-to-treat analysis [26-28]. 2 trials reported data on dropouts [27, 28]. With exception of 1 trial [26], selective reporting was found in almost all of the trials. Baseline similarity was described in all the studies, but 7 trials did not mention the H \& Y stage [18, $19,23,24,30-32] .15$ trials reported constant cointervention, whereas 4 studies were ambiguous $[18,25,30,31]$. All of the included studies appeared to have acceptable adequate compliance and similar timing outcome assessments. In general, 14 RCTs were deemed to have an unclear risk of bias based on the Cochrane Risk of Bias tool, and the remaining 5 trials are high-quality clinical trials [26-29, 35].

\section{Synthesis of Results}

4.1. High-Frequency Herbs Found in TCM Prescriptions for $P D$. Based on our review, we documented and ranked the top 16 individual Chinese herbs for PD treatment that were used more than 3 times in the TCM prescriptions of the 19 included trials (Table 3). For example, Prepared Rehmannia Root, White peony Alba, Szechwan Lovage Rhizome, and Tall 


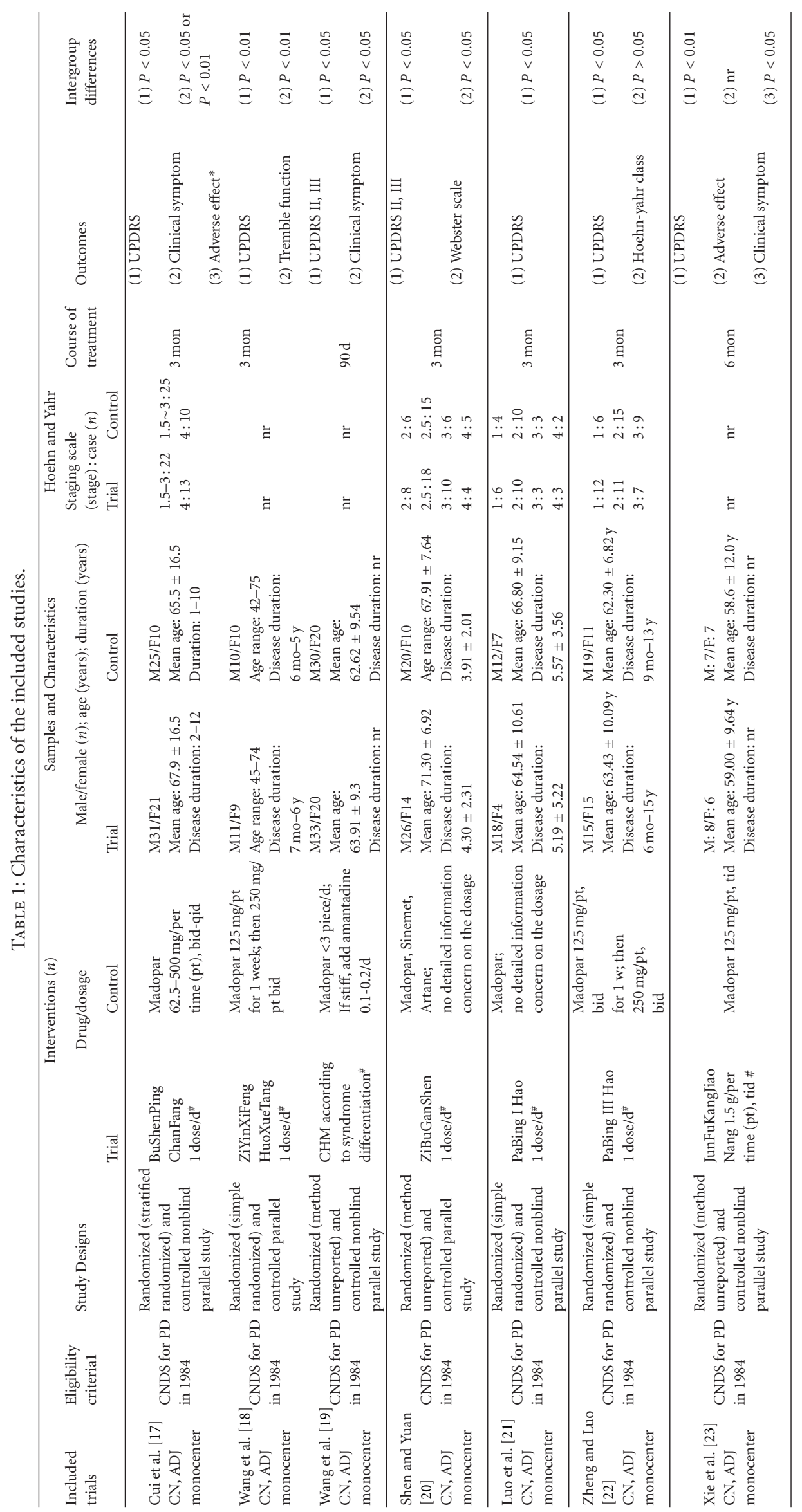




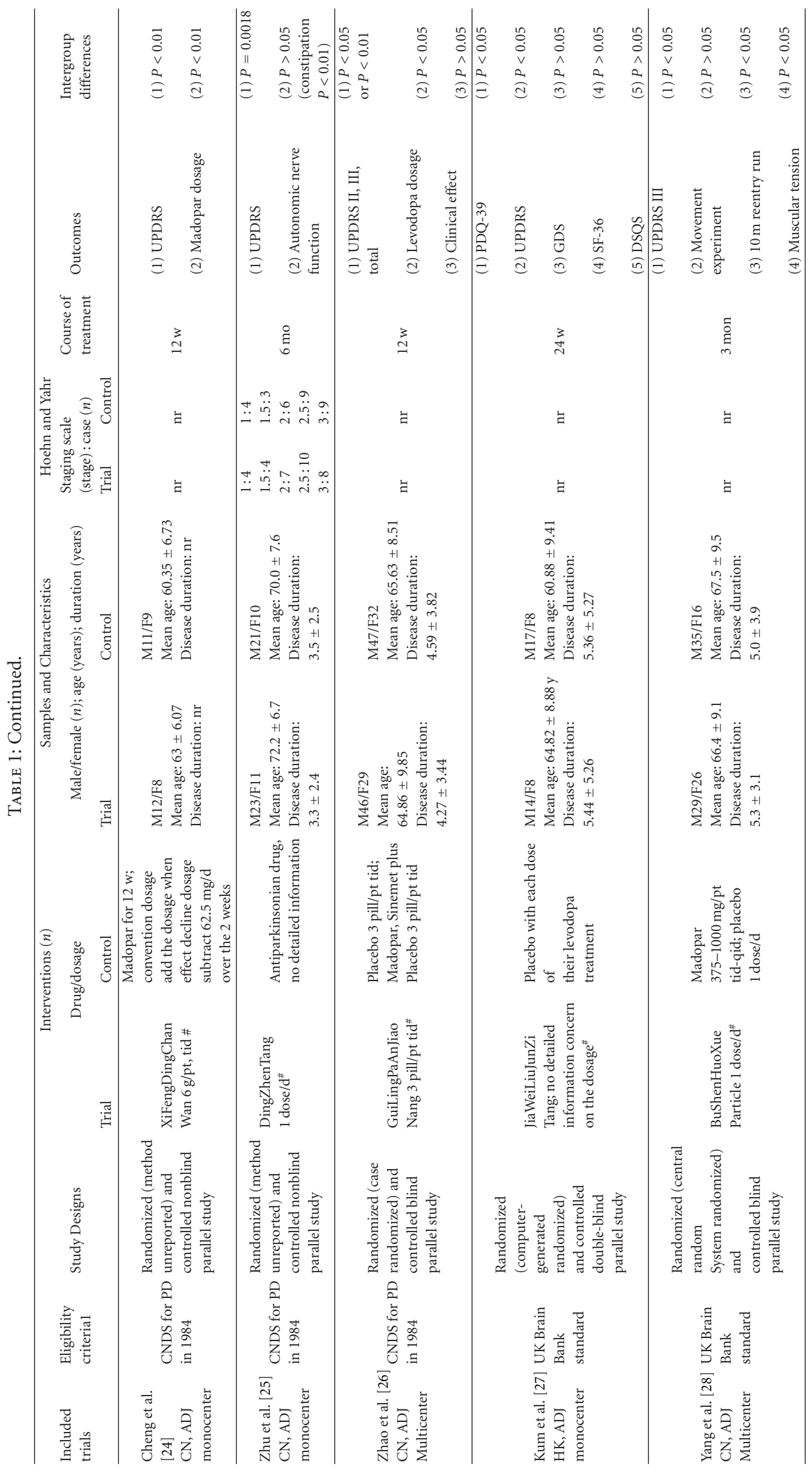




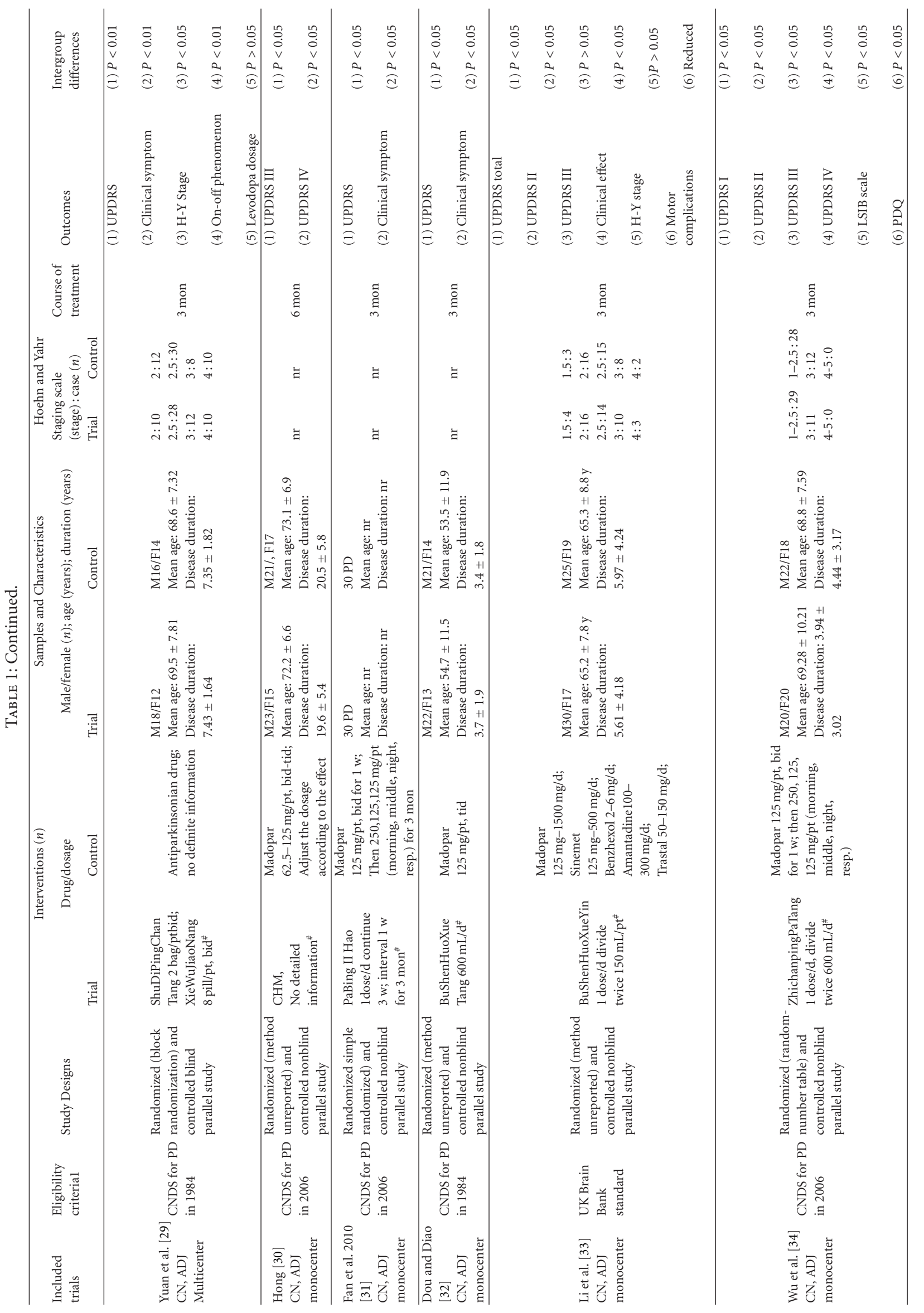




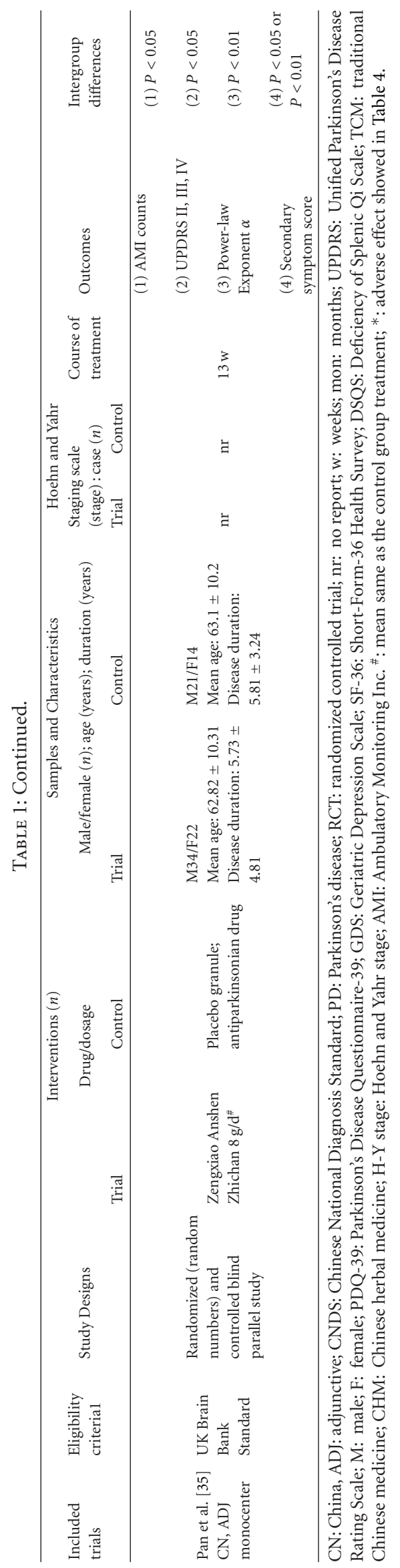


TABLE 2: The included trials scored according to the risk of bias criteria.

\begin{tabular}{|c|c|c|c|c|c|c|c|c|c|c|c|c|c|c|c|}
\hline & $\mathrm{A}$ & $\mathrm{B}$ & $\mathrm{C}$ & $\mathrm{D}$ & $\mathrm{E}$ & $\mathrm{F}$ & G & $\mathrm{H}$ & $\mathrm{I}$ & $\mathrm{J}$ & $\mathrm{K}$ & $\mathrm{L}$ & Total $+/ 12$ & Total $-/ 12$ & Total ?/12 \\
\hline Cui et al. [17] & + & - & - & - & - & $?$ & $?$ & $?$ & + & + & $?$ & + & 4 & 4 & 4 \\
\hline Wang et al. [18] & + & + & - & - & - & ? & $?$ & $?$ & $?$ & $?$ & + & + & 4 & 3 & 5 \\
\hline Wang et al. [19] & $?$ & - & - & - & - & $?$ & $?$ & $?$ & $?$ & + & $?$ & + & 2 & 4 & 6 \\
\hline Shen and Yuan [20] & $?$ & - & - & - & - & $?$ & $?$ & $?$ & + & + & $?$ & + & 3 & 4 & 5 \\
\hline Luo et al. [21] & $?$ & - & - & - & - & $?$ & $?$ & $?$ & + & + & + & + & 4 & 4 & 4 \\
\hline Zheng and Luo [22] & $?$ & - & - & - & - & $?$ & $?$ & ? & + & + & + & + & 4 & 4 & 4 \\
\hline Xie et al. [23] & $?$ & - & - & - & - & $?$ & ? & $?$ & ? & + & + & + & 3 & 4 & 5 \\
\hline Cheng et al. [24] & $?$ & - & - & - & - & $?$ & $?$ & $?$ & $?$ & + & $?$ & + & 2 & 4 & 6 \\
\hline Zhu et al. [25] & $?$ & - & - & - & - & $?$ & ? & $?$ & + & $?$ & $?$ & + & 2 & 4 & 6 \\
\hline Zhao et al. [26] & + & + & + & + & + & + & $?$ & + & + & + & + & + & 11 & 0 & 1 \\
\hline Kum et al. [27] & + & - & + & + & - & + & + & $?$ & + & + & + & + & 9 & 2 & 1 \\
\hline Yang et al. [28] & + & + & + & + & - & + & + & $?$ & + & + & + & + & 10 & 1 & 1 \\
\hline Yuan et al. [29] & + & + & + & + & - & $?$ & $?$ & $?$ & + & $?$ & + & + & 7 & 1 & 4 \\
\hline Hong [30] & $?$ & - & - & - & - & $?$ & $?$ & $?$ & $?$ & + & $?$ & + & 2 & 4 & 6 \\
\hline Fan et al. [31] & - & - & - & - & - & $?$ & $?$ & $?$ & $?$ & + & + & + & 3 & 5 & 4 \\
\hline Dou and Diao [32] & $?$ & - & - & - & - & $?$ & $?$ & $?$ & $?$ & + & + & + & 3 & 4 & 4 \\
\hline Li et al. [33] & $?$ & - & - & - & - & $?$ & $?$ & $?$ & + & + & + & + & 4 & 4 & 4 \\
\hline Wu et al. [34] & + & - & - & - & - & $?$ & $?$ & $?$ & + & + & + & + & 5 & 4 & 3 \\
\hline Pan et al. [35] & + & + & + & + & - & $?$ & + & $?$ & + & $?$ & $?$ & + & 8 & 0 & 4 \\
\hline
\end{tabular}

A: adequate sequence generation; B: concealment of allocation; C: blinding (patient); D: blinding (investigator); E: blinding (assessor); F: incomplete outcome data addressed (ITT analysis); G: incomplete outcome data addressed (dropouts); H: free of selective reporting; I: similarity at baseline; J: cointerventions constant; K: compliance acceptable; L: similar timing outcome assessments. +: yes, -: no, ?: unclear.

Gastrodis Tuber are the top 4 most frequently used herbs. The main effects of these herbs include replenishing blood and tonifying Yin, calming the liver, checking endogenous wind, dispelling evil-wind, and activating blood flow. These high-frequency herbs may contribute in composing a fundamental prescription for clinical PD treatment and seems worthy of additional, indepth study.

4.2. UPDRS I Scores. The 5 independent trials showed the homogeneity in the consistency of the trial results, chi-square $=3.69(P=0.45) ; I^{2}=0 \%$. Thus, fixed-effects model should be used for statistical analysis. Compared to conventional treatment, CHM paratherapy significantly improved UPDRS I scores (WMD $-0.33,95 \% \mathrm{CI}-0.58$ to $-0.08 ; Z=2.60(P<$ $0.001)$ ). The difference suggested that CHM paratherapy was more effective than conventional treatment for symptoms of mentation, behavior, and mood in patients with PD (Table 4). The funnel plot was roughly symmetric. There would be little publication bias for the 5 independent trials (Figure 2).

4.3. UPDRS II Scores. The 9 independent literatures showed homogeneity in the results of trials, chi-square $=3.26(P=$ 0.92); $I^{2}=0 \%$. Thus, fixed-effects model should be used for statistical analysis. Compared to conventional treatment, CHM paratherapy significantly improved UPDRS II scores (WMD $-2.18,95 \% \mathrm{CI}-3.03$ to $-1.33 ; Z=5.03(P<$ $0.001)$ ), suggesting that CHM paratherapy could contribute to improving the activities of daily life (ADLs) in patients with PD (Table 5). The funnel plot was symmetric. No evidence of publication bias was found (Figure 3 ).

4.4. UPDRS III Scores. The 12 independent trials did not show homogeneity in the trial results, chi-square $=89.22$, $(P<0.001) ; I^{2}=88 \%$. Thus, random-effects model should be used for statistical analysis. Compared to conventional treatment, CHM paratherapy significantly improved UPDRS III scores (WMD $-2.35,95 \% \mathrm{CI}-4.61$ to $-0.08 ; Z=2.03$ $(P<0.05))$. This result suggested that $\mathrm{CHM}$ paratherapy could contribute to improving motor function in patients with PD (Table 6). The funnel plot was markedly asymmetric. There exists a publication bias in the 12 independent trials (Figure 4).

4.5. UPDRS IV Scores. The 7 independent studies showed homogeneity in the trial results, chi-square $=5.21(P=$ $0.52) ; I^{2}=0 \%$. Thus, fixed-effects model should be used for statistical analysis. Compared to conventional treatment, CHM paratherapy significantly improved UPDRS IV scores (WMD $-0.51,95 \% \mathrm{CI}-0.83$ to $-0.20 ; Z=3.61(P<$ $0.05)$ ), suggesting that CHM paratherapy could contribute to improving complications of treatment in patients with $\mathrm{PD}$ (Table 7). The funnel plot was obviously asymmetric. There exists a publication bias in the 7 independent trials with mainly positive results (Figure 5).

4.6. UPDRS I-IV Total Summed Score. The 10 independent trials showed homogeneity in the trial results, chi-square $=$ $4.25(P=0.89) ; I^{2}=0 \%$. Thus, fixed-effects model should 
TABLE 3: The 16 herbs used more than 3 times for PD in the 19 trials included.

\begin{tabular}{|c|c|c|c|c|c|}
\hline Chinese Pinyin & Latin herb name & English herb name & Frequency & The total frequency (127)\% & Dosage \\
\hline Dihuang & Radix Rehmanniae preparata & Prepared Rehmannia Root & 10 & 7.9 & $10-24 \mathrm{~g}$ \\
\hline Baishao & Radix Paeoniae Alba & White peony Alba & 10 & 7.9 & $12-30 \mathrm{~g}$ \\
\hline Chuanxiong & Rhizoma Chuanxiong & Szechwan Lovage Rhizome & 10 & 7.9 & $12-15 \mathrm{~g}$ \\
\hline Tianma & Rhizoma Gastrodiae & Tall Gastrodis Tuber & 9 & 7.1 & $10-20 \mathrm{~g}$ \\
\hline Gouteng & Ramulus Uncariae Cum Uncis & Gambir Plant & 6 & 4.7 & $15-20 \mathrm{~g}$ \\
\hline Danggui & Radix Angelicae Sinensis & Chinese Angelica & 6 & 4.7 & $10-20 \mathrm{~g}$ \\
\hline Heshouwu & Radix Polygoni Multiflori & Fleeceflower Root & 5 & 3.9 & $15-20 \mathrm{~g}$ \\
\hline Shanzhuyu & Fructus Corni & Asiatic Cornelian Cherry Fruit & 5 & 3.9 & $8-20 \mathrm{~g}$ \\
\hline Shichangpu & Rhizoma Acori Tatarinowii & Grassleaf Sweetflag Rhizome & 4 & 3.1 & $10 \mathrm{~g}$ \\
\hline Quanxie & Scorpio & Scorpion & 4 & 3.1 & $1.5-10 \mathrm{~g}$ \\
\hline Jiangcan & Bombyx Batryticatus & Stiff Silkorm & 4 & 3.1 & $9-15 \mathrm{~g}$ \\
\hline Danshen & Radix Salviae Miltiorrhizae & Danshen Root & 4 & 3.1 & $10-15 \mathrm{~g}$ \\
\hline Wumei & Fructus Mume & Smoked Plum & 4 & 3.1 & $9-15 \mathrm{~g}$ \\
\hline Huanglian & Rhizoma Coptidis & Golden Thread & 3 & 2.4 & $9-15 \mathrm{~g}$ \\
\hline Roucongrong & Herba Cistanches & Desertliving Cistanche & 3 & 2.4 & $10-15 \mathrm{~g}$ \\
\hline Tiannanxing & Rhizoma Arisaematis & Jackinthepulpit Tuber & 3 & 2.4 & $10-15 \mathrm{~g}$ \\
\hline
\end{tabular}

TABLE 4: Forest plot of comparison: Chinese herbal medicine versus conventional treatment: UPDRS I scores.

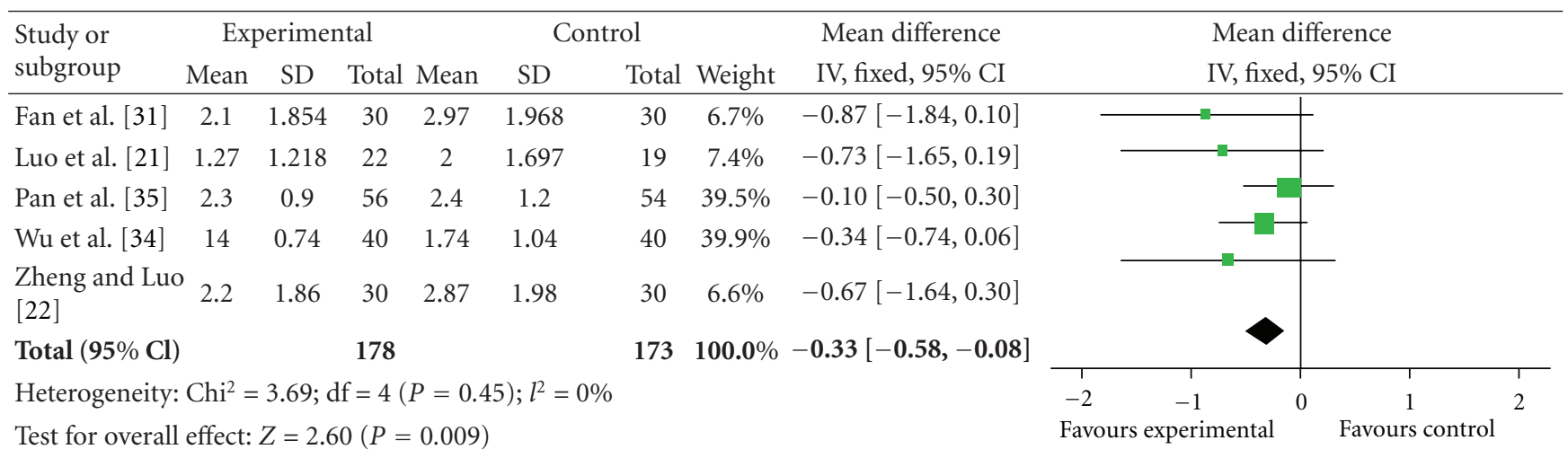

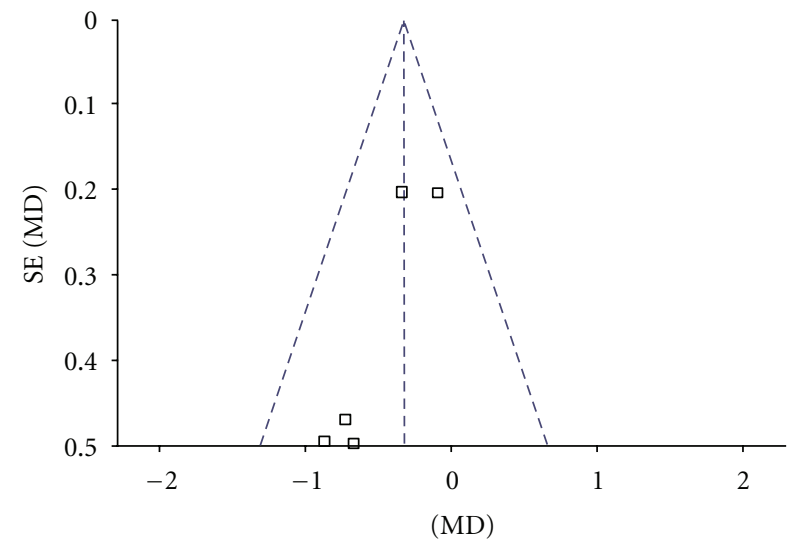

FIGURE 2: Funnel plot of comparison: Chinese herbal medicine versus conventional treatment: UPDRS I scores.

be used for statistical analysis. Compared to conventional treatment, CHM paratherapy significantly improved UPDRS I-IV total summed score (WMD $-6.09,95 \%$ CI -8.08 to
$-4.10 ; Z=6.00(P<0.001))$, suggesting that $\mathrm{CHM}$ paratherapy could contribute to improving symptoms of $\mathrm{PD}$ (Table 8). The funnel plot showed nearly complete symmetry. No publication bias was found in the 10 independent trials included (Figure 6).

4.7. Adverse Effects. Adverse effects were reported in 10 studies [17, 20, 22-24, 27-29, 32, 35], but no mention of side effects in the other 9 trials was reported (Table 9). There were no significant differences in the results of blood routine, urine routine, liver function, renal function, or electrocardiograph (ECG) in both groups of patients before and after treatment $[20,22,28]$. Diarrhea $[27,28]$, constipation $[20,23,29]$, nausea and/or vomiting [17, 20, $23,24,29,32]$, dry mouth $[17,20]$, and dizziness [17, $23,24]$ were reported in CHM paratherapy group. Other adverse effects including arrhythmia [24], epigastric pain [29], sialorrhea, hypotension, insomnia, and depression [32] were reported. However, no life-threatening adverse effects were noted in these studies, and the side effects were less than or lighter than the conventional treatment group. 
TABLE 5: Forest plot of comparison: Chinese herbal medicine versus conventional treatment: UPDRS II scores.

\begin{tabular}{lcccccccccc}
\hline $\begin{array}{l}\text { Study or } \\
\text { subgroup }\end{array}$ & Mean & SD & Total & Mean & SD & Total & Weight & lV, fixed, 95\% Cl & Mean difference \\
lV, fixed, 95\% Cl
\end{tabular}

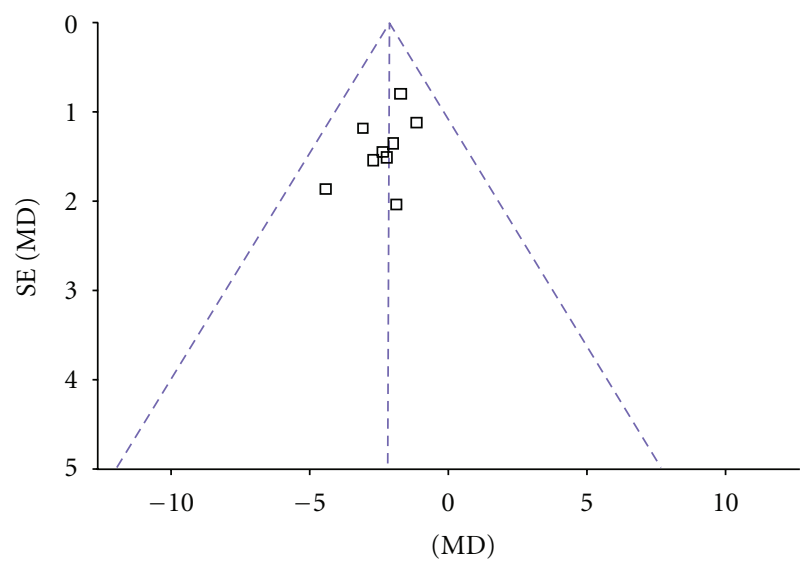

FIGURE 3: Funnel plot of comparison: Chinese herbal medicine versus conventional treatment: UPDRS II scores.

\section{Discussion}

5.1. Summary of Evidence. The main findings of this metaanalysis were that CHM adjuvant therapy could improve the clinical symptom severity scores for PD and has few adverse effects in comparison to WCM controls. The evidences of CHM paratherapy for PD are emerging and the evidences presented in this meta-analysis potentially benefit a clinical recommendation in spite of some methodological weaknesses. However, there was still not enough replicable evidence to conclude that any specific CHM therapy is effective for WD.

The CHMs evaluated in this paper generally appeared to be safe and well tolerated in patients with PD. However, the safety for the use of CHMs could not be confirmed because only $47.37 \%$ (9/19) studies mentioned the safety of interventions or investigated adverse effects. It is recommended that more attention should be given to both recording and reporting the adverse effects of these interventions.

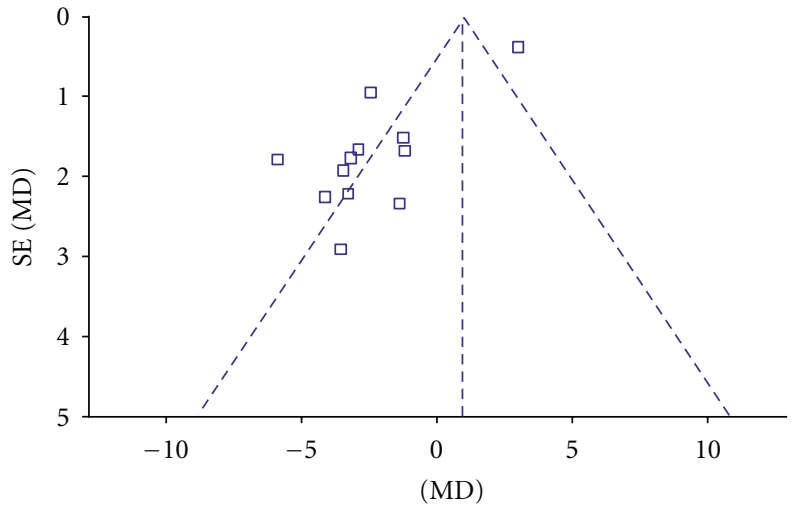

FIGURE 4: Funnel plot of comparison: Chinese herbal medicine versus conventional treatment: UPDRS III scores.

5.2. Limitations. There are a number of inherent and methodological limitations to this meta-analysis. First of all, none of included studies had been registered. In September 2004, the members of the International Committee of Medical Journal Editors (ICMJE) published a statement requiring that all clinical trials must be registered in order to be considered for publication [36]. Clinical trial registration will improve research transparency and will ultimately strengthen the validity and value of the scientific evidence base. Thus, the inherent limitation of this paper existed in the primary studies.

One of the major limitations was the application of various kinds of CHMs add-on therapy used in different trials. They differ in composition, dosage preparation, and methods and manufacturing standards. It is difficult to assess the effect of a particular CHM by means of the evidence synthesis of studies.

There are many methodological weaknesses in this metaanalysis. (1) Randomization: all included studies claimed randomization. However, only $8 / 19$ trials provided sufficient 
TABLE 6: Forest plot of comparison: Chinese herbal medicine versus conventional treatment: UPDRS III scores.

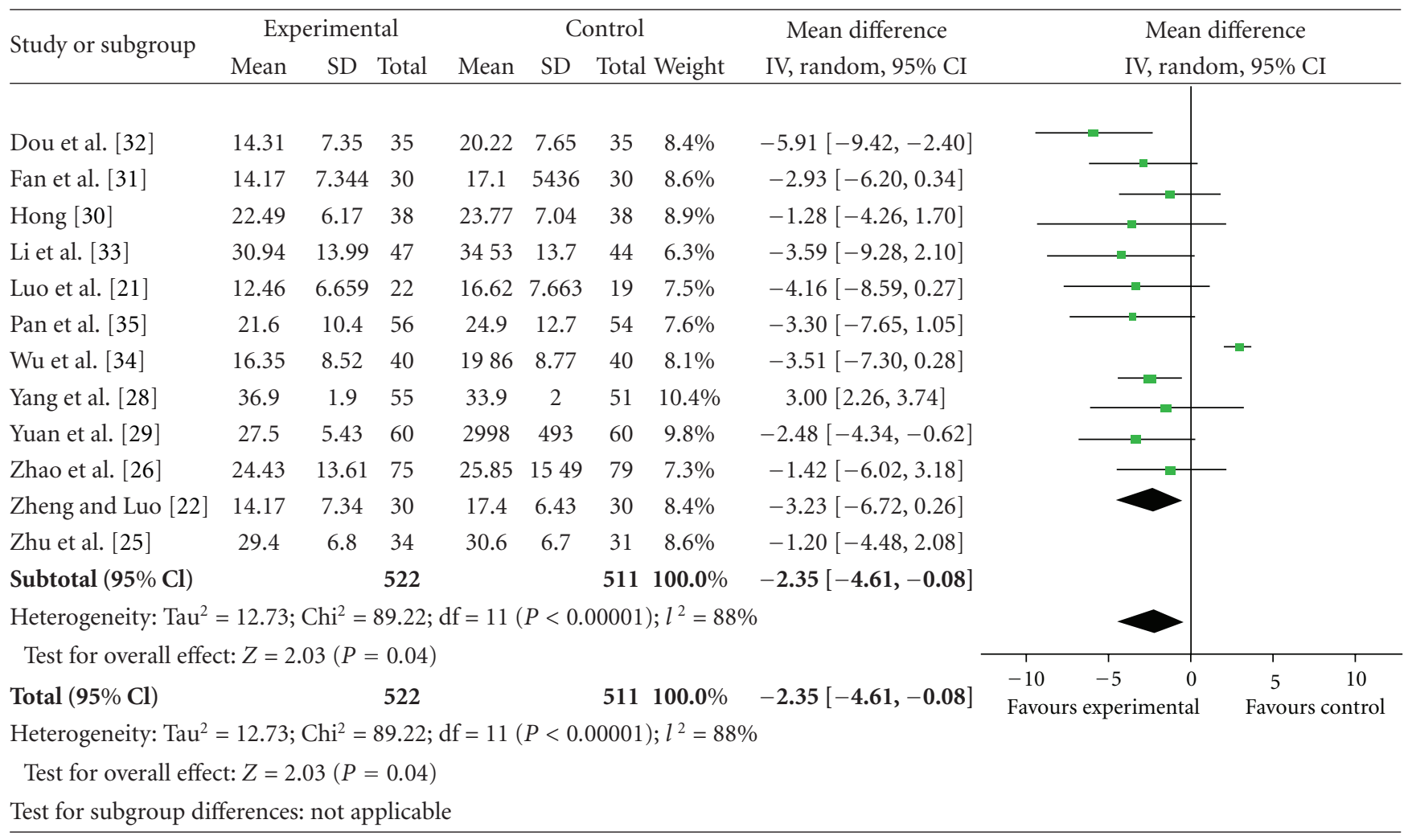

TABLE 7: Forest plot of comparison: Chinese herbal medicine versus conventional treatment: UPDRS IV scores.

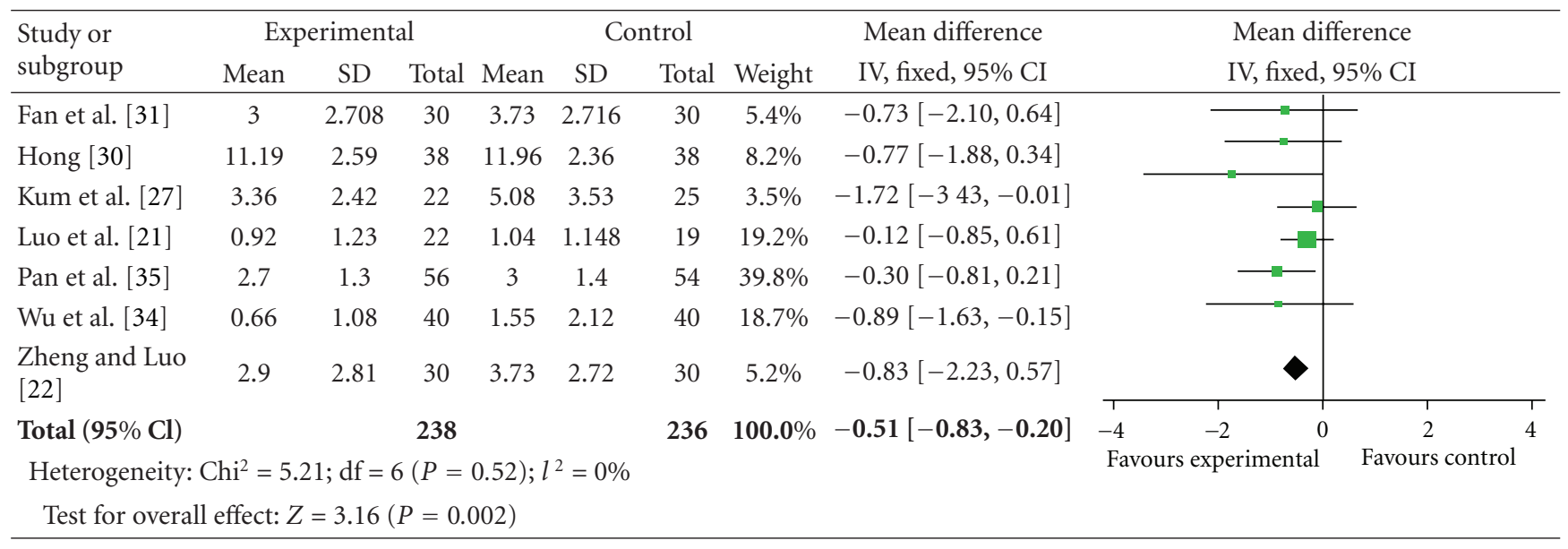

information on how the random allocation was generated such as from random-number table, calculator or computer random-number generator; 5/19 trials reported allocation concealment such as sealed envelopes or a telephone call to the research centre. The proper randomization in RCTs is necessary to avoid selection bias and confounding. Thus, an invalid method of randomization could have distorted our results. (2) Blinding: with exception of blinding (participants and care providers) in 4 trials, the other 15 studies were lack of any blinding method which can produce performance bias and detection bias. Blinding of the outcome assessor was only used in one study. Thus, assessment of outcomes was prone to significant systemic errors. (3) Analysis of data from RCTs: dropouts were only reported in 3 trials, and 1 trial of intention-to-treat analysis was mentioned. Therefore, the results generated from these studies should be interpreted with caution. (4) Placebo controlled: only 4 trials out of the 19 included studies have placebo control. The other 15 trials used an "A + B versus B" design where patients were randomized to receive a $\mathrm{CMH}$ paratherapy plus WCM 
TABLE 8: Forest plot of comparison: Chinese herbal medicine versus conventional treatment: UPDRS I-IV total scores.

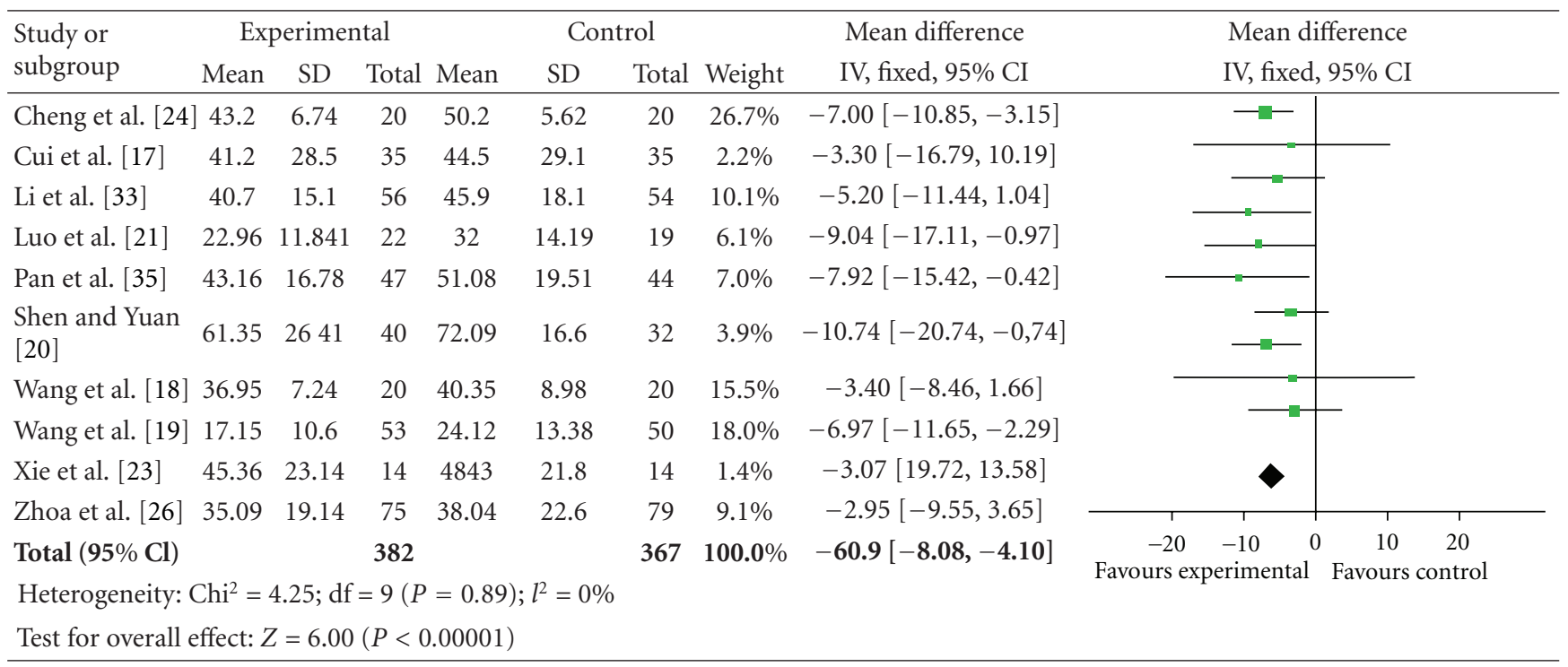

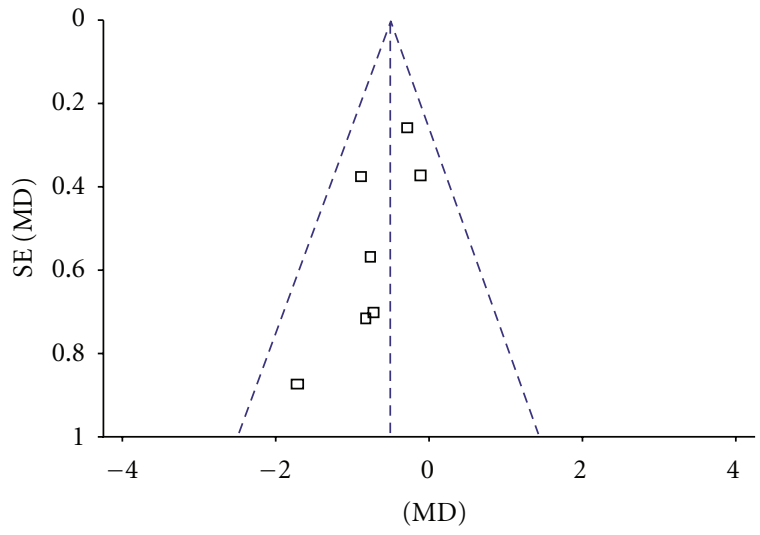

FIGURE 5: Funnel plot of comparison: Chinese herbal medicine versus conventional treatment: UPDRS IV scores.

control treatment versus WCM control treatment without a rigorous control for placebo effect. Thus, the results of these studies would be positive because of nonspecific placebo effects [37]. (5) Sample size: the included studies were of relatively small sample sizes in individual trials and without formal sample size calculation. Trials that lacked proper sample size estimation placed their statistical analysis's validity in doubt. The results were likely to be underpowered. (6) Heterogeneity: the imbalance in gender, ethnicity, and wide range in disease duration further compromised the validity of the included trials. Furthermore, outcome measures used in the trials were heterogeneous and incomplete. Thus, the results might have been compromised by the heterogeneity within each CHM intervention and by the study design.

Another limitation was publication bias. Publication bias was assessed by visual inspection of funnel plots. There was bias with UPDRS III and IV score in CHM paratherapy plus

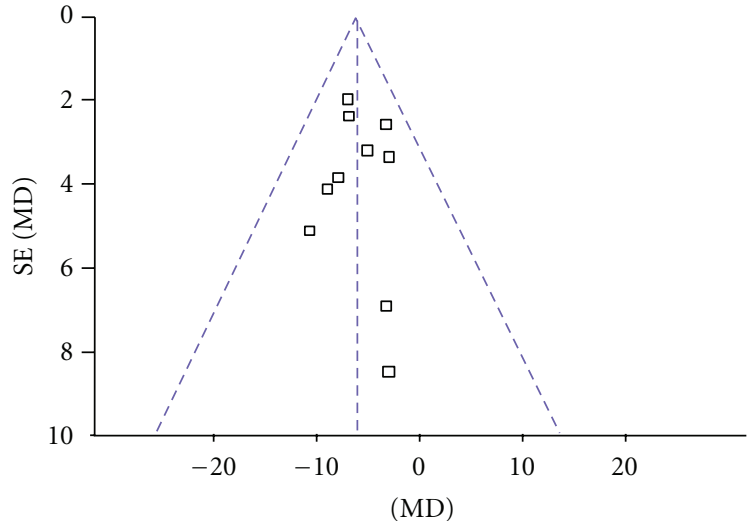

FIGURE 6: Funnel plot of comparison: Chinese herbal medicine versus conventional treatment: UPDRS I-IV total scores.

conventional treatment compared to conventional treatment alone. In a total of 19 studies, results were all positive in CHM paratherapy group. Therefore, the validity of inferences that can be drawn is threatened.

\section{Conclusions}

6.1. Implications for Practice. This is the first meta-analysis of randomized, controlled trials to assess the efficacy and safety of CHM paratherapy in patients with PD. In our metaanalysis, patients receiving CHM adjunct therapy plus WCM exhibit significant improvement in their PD symptoms as evidenced by improvements in their UPDRS scores compared to WCM controls in spite of some methodological limitations. According to the safety assessment of this metaanalysis, the CHM add-on therapy for PD is generally safe 
TABLE 9: Adverse effects found in CHMs for PD in the 19 trials included.

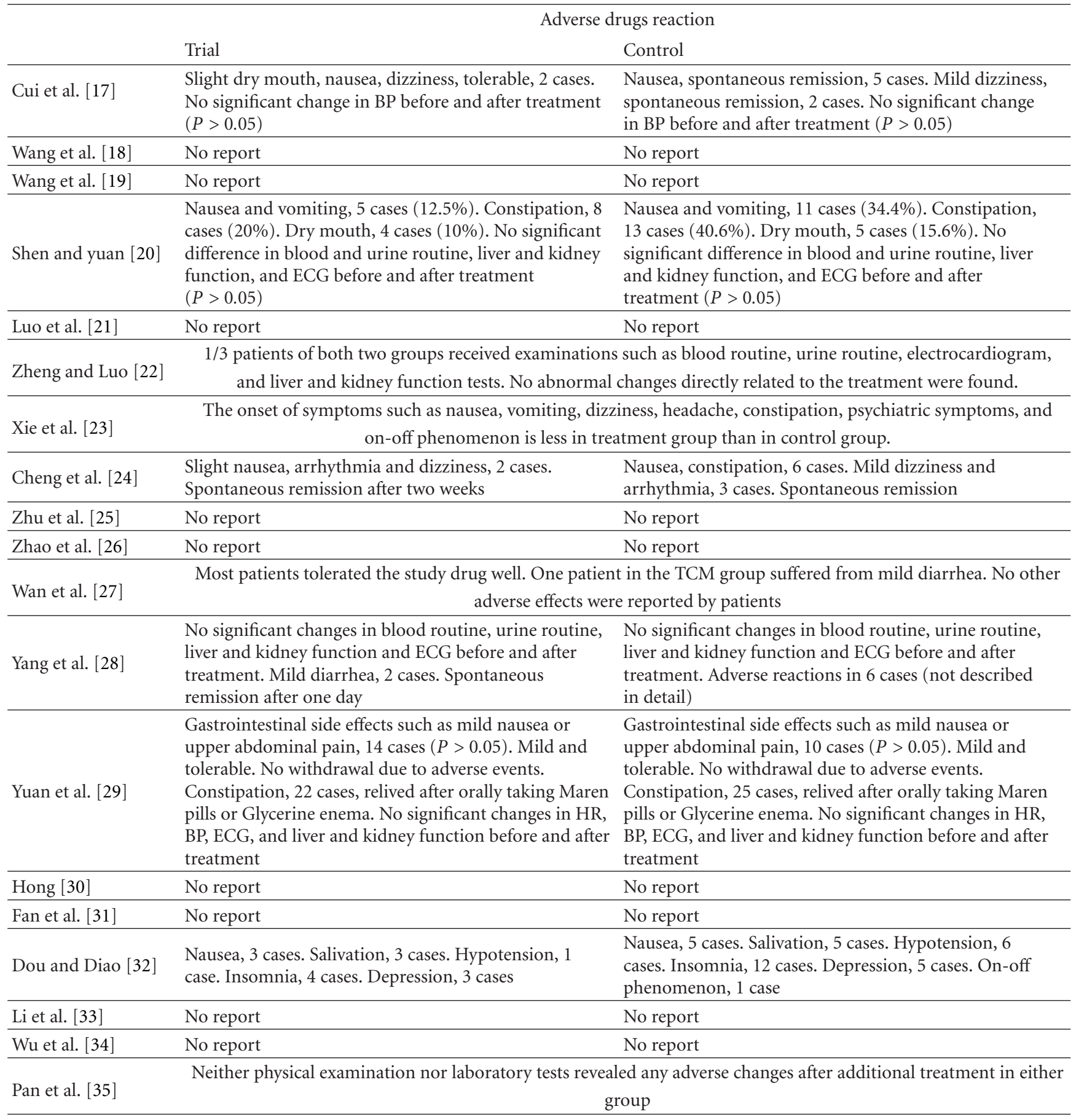

BP: blood pressure; ECG: electrocardiography; HR: heart rate.

and well tolerated. Therefore, CHM paratherapy may be effective and well tolerated for the symptomatic treatment of PD. However, various kinds of CHMs paratherapy were used in different trials. As such, treatment choices must be consider each individual's CHM. Although acknowledging the limitations of this meta-analysis, our findings present several high-quality trials $[26-29,35]$ and provide potential evidences that CHM adjunct therapy can additionally benefit relieve symptoms of $\mathrm{PD}$. However, methodological robust trials are still needed to further evaluate this therapy due to the generally low methodological quality of the included studies.

6.2. Implications for Research. A number of implications for research arise from this paper. First, improvement in the methodological quality of randomized controlled trials is critical for later trials and more methodologically rigorous studies are needed in this field. Second, the included trials 
were generally of small sample size. None of the trials reported the method of sample size determination. Sample size calculation should be conducted before enrollment. Third, two ways are performed globally for clinical trial transparency: (1) all clinical trials must be registered before the enrollment of the first patient, based on ICMJE statement; (2) the making and dissemination and implementation of reporting standards of clinical trial represented by CONSORT [38] series. In China, CONSORT for TCM was developed by Wu et al. [39] in 2007. Further well-designed, randomized, double-blind, placebo-controlled trials need to be carried out and reported in detail according to CONSORT or CONSORT for TCM. Fourth, various kinds of different forms of CHMs were tested in the 19 studies included, without detailed information on composition, dosage preparation, and manufacturing standards, and so forth. Thus, it is necessary to identify which one of the herbs displays an anti-Parkinsonian action and find the active component of this herb medicine. In this way, we can assess the effect of a particular CHM by means of the evidence synthesis of trials.

\section{Conflict of Interests}

The authors declared that they have no conflict of interests.

\section{Acknowledgments}

This work is supported by the Development Funding of Zhejiang Extremely Key Subject of Pharmacology and Biochemical Pharmaceutics; the Project of Wenzhou Municipal Science and Technology Bureau in Zhejiang province (H20070044, Y20080145); Administration of Traditional Chinese Medicine of Zhejiang Province (2010ZQ010); Xinmiao Planning Project of Science and Technology Department of Zhejiang Province (2008R40G2090034).

\section{References}

[1] S. S. Rao, L. A. Hofmann, and A. Shakil, "Parkinson's disease: diagnosis and treatment," American Family Physician, vol. 74, no. 12, pp. 2046-2054, 2006.

[2] M. Löhle and H. Reichmann, "Clinical neuroprotection in Parkinson's disease-still waiting for the breakthrough," Journal of the Neurological Sciences, vol. 289, no. 1-2, pp. 104114, 2010.

[3] P. Calabresi, M. D. Filippo, V. Ghiglieri, N. Tambasco, and B. Picconi, "Levodopa-induced dyskinesias in patients with Parkinson's disease: filling the bench-to-bedside gap," The Lancet Neurology, vol. 9, no. 11, pp. 1106-1117, 2010.

[4] T. A. Zesiewicz and M. L. Evatt, "Potential influences of complementary therapy on motor and non-motor complications in parkinsons disease," CNS Drugs, vol. 23, no. 10, pp. 817$835,2009$.

[5] Z. X. Zhang, G. C. Roman, Z. Hong et al., "Parkinson's disease in China: prevalence in Beijing, Xian, and Shanghai," The Lancet, vol. 365, no. 9459, pp. 595-597, 2005.

[6] Y. Wang, X. M. Lin, and G. Q. Zheng, "Traditional Chinese medicine for Parkinson's disease in china and beyond," Journal of Alternative and Complementary Medicine, vol. 17, no. 5, pp. 385-388, 2011.
[7] G. Q. Zheng, “Therapeutic history of Parkinson's disease in Chinese medical treatises," Journal of Alternative and Complementary Medicine, vol. 15, no. 11, pp. 1223-1230, 2009.

[8] Y. C. Lam, W. F. Kum, S. S. K. Durairajan et al., "Efficacy and safety of acupuncture for idiopathic Parkinson's disease: a systematic review," Journal of Alternative and Complementary Medicine, vol. 14, no. 6, pp. 663-671, 2008.

[9] Q. Li, D. Zhao, and E. Bezard, "Traditional Chinese medicine for Parkinson's disease: a review of Chinese literature," Behavioural Pharmacology, vol. 17, no. 5-6, pp. 403-410, 2006.

[10] V. Chung, L. Liu, Z. Bian et al., "Efficacy and safety of herbal medicines for idiopathic Parkinson's disease: a systematic review," Movement Disorders, vol. 21, no. 10, pp. 1709-1715, 2006.

[11] D. Moher, A. Liberati, J. Tetzlaff, and D. G. Altman, "Preferred reporting items for systematic reviews and meta-analyses: the PRISMA statement," PLoS Medicine, vol. 6, Article ID e1000097, 2009.

[12] A. J. Hughes, S. E. Daniel, L. Kilford, and A. J. Lees, "Accuracy of clinical diagnosis of idiopathic Parkinson's disease: a clinico-pathological study of 100 cases," Journal of Neurology Neurosurgery and Psychiatry, vol. 55, no. 3, pp. 181-184, 1992.

[13] X. D. Wang, "Standard for diagnosing and distinguishing Parkinson's disease and Parkinsonian syndromes," Chinese Journal of Nervous and Mental Disease, vol. 18, pp. 256-257, 1985 (Chinese).

[14] The Writing Group of the Movement Disorders and Parkinson's Disease Society of Neurology Committee of Chinese Medical Association, "The diagnosis of Parkinson's disease," Chinese Journal of Neurology, vol. 39, pp. 408-409, 2006.

[15] S. Fahn, R. L. Elton, and U. D. Committee, "Unified Parkinson," in Recent Developments in Parkinson's Disease, S. Fahn, C. D. Marsden, and D. B. Calne, Eds., vol. 2, pp. 153-173, Macmillan Healthcare Information, Florham Park, NJ, USA, 1987.

[16] A. D. Furlan, V. Pennick, C. Bombardier, and M. Van Tulder, "2009 Updated method guidelines for systematic reviews in the cochrane back review group," Spine, vol. 34, no. 18, pp. 1929-1941, 2009.

[17] L. Cui, X. D. Yu, and J. Cui, "Clinical observation on effect of combined therapy of Bushenpingchan recipe and medopa tablet in treating Parkinson's disease," Chinese Journal of Integrated Traditional and Western Medicine, vol. 23, pp. 504507, 2003 (Chinese).

[18] W. T. Wang, X. D. Luo, W. Wu, C. Y. Zheng, and Y. Z. Sun, "Clinical observation on 20 cases of early stage of Parkinson's disease with Ziyin Xifeng Huoxue decoction," Journal of Traditional Chinese Medicine, vol. 45, pp. 274-275, 2004 (Chinese).

[19] Z. Y. Wang, Y. L. Zhao, Y. J. Li, and H. M. Li, "Clinical observation of integrated traditional Chinese and western medicine in treating Parkinson's disease," Capital Medicine, vol. 12, pp. 32-33, 2005 (Chinese).

[20] W. Shen and C. X. Yuan, "Clinical observation on treatment of Parkinson's disease by nourishing liver and kidney," Shanghai Journal of Traditional Chinese Medicine, vol. 40, pp. 21-22, 2006 (Chinese).

[21] X. D. Luo, Y. Z. Sun, and X. F. Lian, "Clinical experiment study of first pabing prescription on treating stiffing palsy," Journal of Traditional Chinese Medicine, vol. 2, p. 148, 2006 (Chinese).

[22] X. D. Zheng and C. Y. Luo, "Clinical study on pabing recipe for treatment of 30 cases of Parkinson's disease," Journal of Traditional Chinese Medicine, vol. 47, pp. 516-517, 2006 (Chinese). 
[23] X. J. Xie, S. W. Jia, and Z. M. Wu, "Clinical observation on 14 cases of Parkinson's disease with a combined treatment of Junfukang Capsule and madopar," Journal of New Chinese Medicine, vol. 38, pp. 45-46, 2006 (Chinese).

[24] C. H. Cheng, Y. Z. Ma, L. Ma, and J. F. Wang, "Study on the effect of Xifeng Dingchan Pill on the madopar dosage on Parkinson's disease," Shaanxi Journal of Traditional Chinese Medicine, vol. 23, pp. 35-36, 2007 (Chinese).

[25] X. Y. Zhu, Z. L. Fang, Y. Liu, and C. S. Li, "Clinical trial treatment of Parkinson's disease with Dingzhentang," Journal of Neurology and Neurorehabilitation, vol. 6, pp. 114-116, 2009 (Chinese).

[26] G. H. Zhao, Q. G. Meng, and X. D. Yu, "A multi-centered randomized double-blinded controlled clinical study on efficacy of Gulling Paan Capsule in treating Parkinson's disease," Chinese Journal of Integrated Traditional and Western Medicine, vol. 29, pp. 590-594, 2009 (Chinese).

[27] W. F. Kum, S. S. Durairajan, and Z. X. Bian, "Treatment of idiopathic Parkinson's disease with traditional Chinese herbal medicine: a randomized placebo-controlled pilot clinical study," Evidence-Based Complementary and Alternative Medicine, vol. 2011, Article ID 724353, 8 pages, 2011.

[28] M. H. Yang, M. Li, Y. Q. Dou et al., "Effects of Bushen Huoxue Granule on motor function in patients with Parkinson's disease: a multicenter, randomized, double-blind and placebocontrolled trial," Journal of Chinese Integrative Medicine, vol. 8, no. 3, pp. 231-237, 2010.

[29] C. X. Yuan, H. P. Zhi, S. Z. Chen et al., "Clinical multicenter randomized controlled study on treatment of Parkinson's disease with Shudi Pingchan decoction and western medicine," Shanghai Journal of Traditional Chinese Medicine, vol. 44, pp. 3-6, 2010 (Chinese).

[30] X. Hong, "The observation of effect of integrated traditional Chinese medicine and western medicine for treatment of 38 cases old patients with Parkinson's disease," Shenzhen Journal of Integrated Traditional Chinese and Western Medicine, vol. 20, pp. 168-170, 2010 (Chinese).

[31] Y. P. Fan, L. Zeng, Y. Z. Sun, Q. Z. Su, and X. F. Lian, "Clinical experiment study of second pabing prescription on treating tremor-type of Parkinson's disease," Tianjin Journal of Traditional Chinese Medicine, vol. 27, pp. 190-191, 2010 (Chinese).

[32] L. M. Dou WHDiao, "Clinical study on treatment of Parkinson's disease with Bushen Huoxue decoction," Journal of Changchun University of Traditional Chinese Medicine, vol. 26, pp. 501-502, 2010 (Chinese).

[33] J. Y. Li, M. H. Yang, Y. Q. Dou, Y. Liu, and M. Li, "Clinical effect of Bushenhuoxueyin on patients with Parkinson's disease," Medical Journal of Chinese People's Liberation Army, vol. 36, pp. 273-276, 2011 (Chinese).

[34] L. Wu, X. H. Xu, W. Chen, and P. Li, "The clinical research on standardization of Parkinson's disease treated with a comprehensive treatment program of traditional Chinese and western medicine," Liaoning Journal of Traditional Chinese Medicine, vol. 38, pp. 313-316, 2011 (Chinese).

[35] W. Pan, S. Kwak, Y. Liu et al., "Traditional chinese medicine improves activities of daily living in parkinson's disease," Parkinson's Disease, Article ID 789506, 2011.

[36] C. De Angelis, J. M. Drazen, F. A. Frizelle et al., "Clinical trial registration: a statement from the international committee of medical journal editors," The New England Journal of Medicine, vol. 351, no. 12, pp. 1250-1251, 2004.
[37] E. Ernst and M. Lee, "A trial design that generates only "positive" results," Journal of Postgraduate Medicine, vol. 54, no. 3, pp. 214-216, 2008.

[38] K. F. Schulz, D. G. Altman, and D. Moher, "CONSORT 2010 statement: updated guidelines for reporting parallel group randomised trials," PLoS Medicine, vol. 7, no. 3, Article ID e1000251, 2010

[39] T. X. Wu, Y. P. Li, Z. X. Bian et al., "Consolidated standards for reporting trials of traditional Chinese medicine (CONSORT for TCM) (for solicitation of comments)," Chinese Journal of Evidence-Based Medicine, vol. 7, no. 9, pp. 625-630, 2007. 


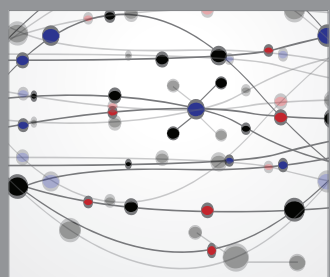

The Scientific World Journal
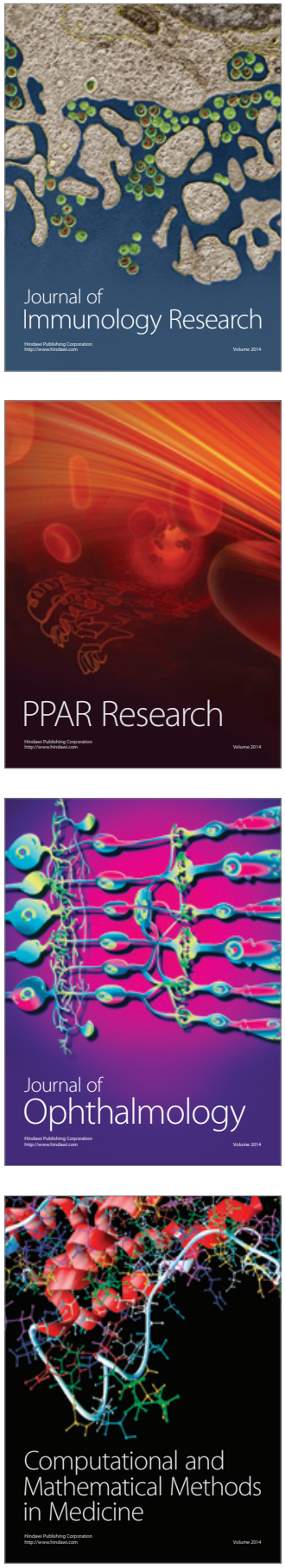

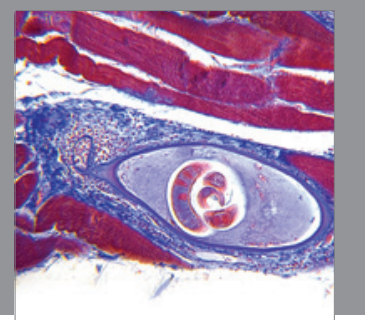

Gastroenterology

Research and Practice
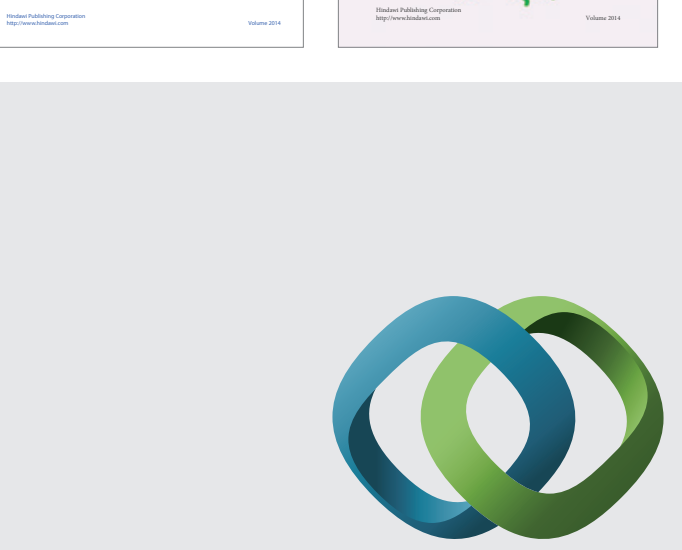

\section{Hindawi}

Submit your manuscripts at

http://www.hindawi.com
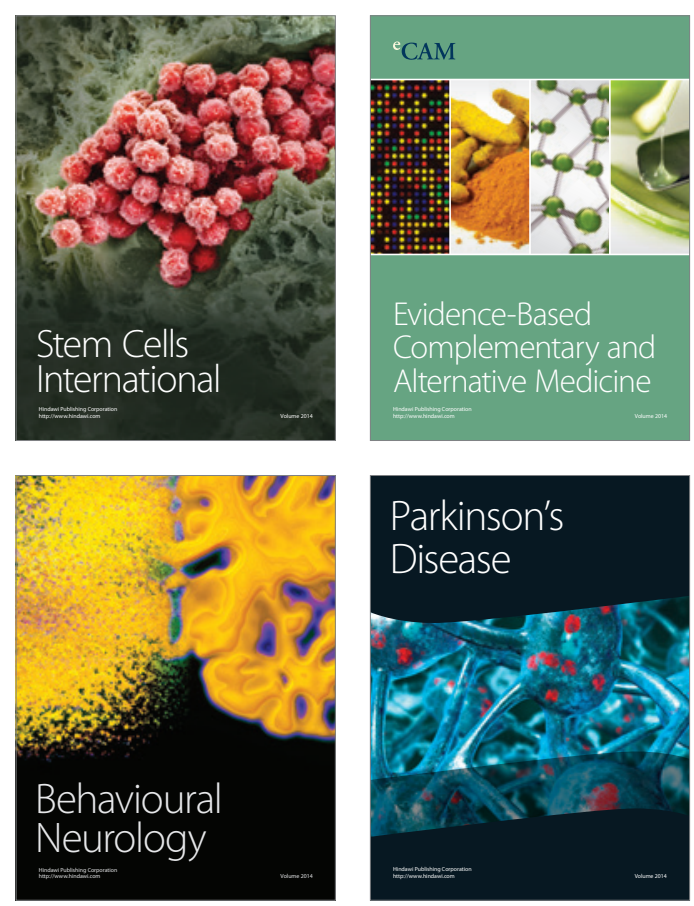

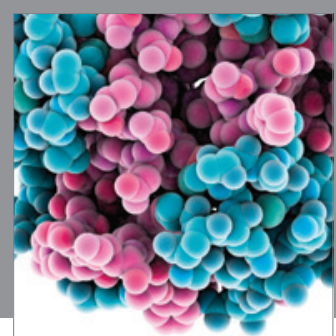

Journal of
Diabetes Research

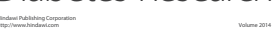

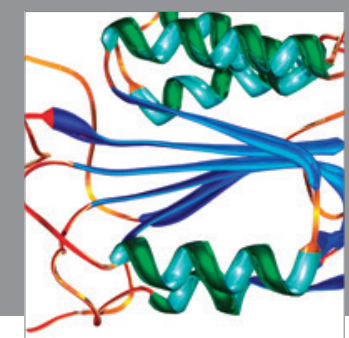

Disease Markers
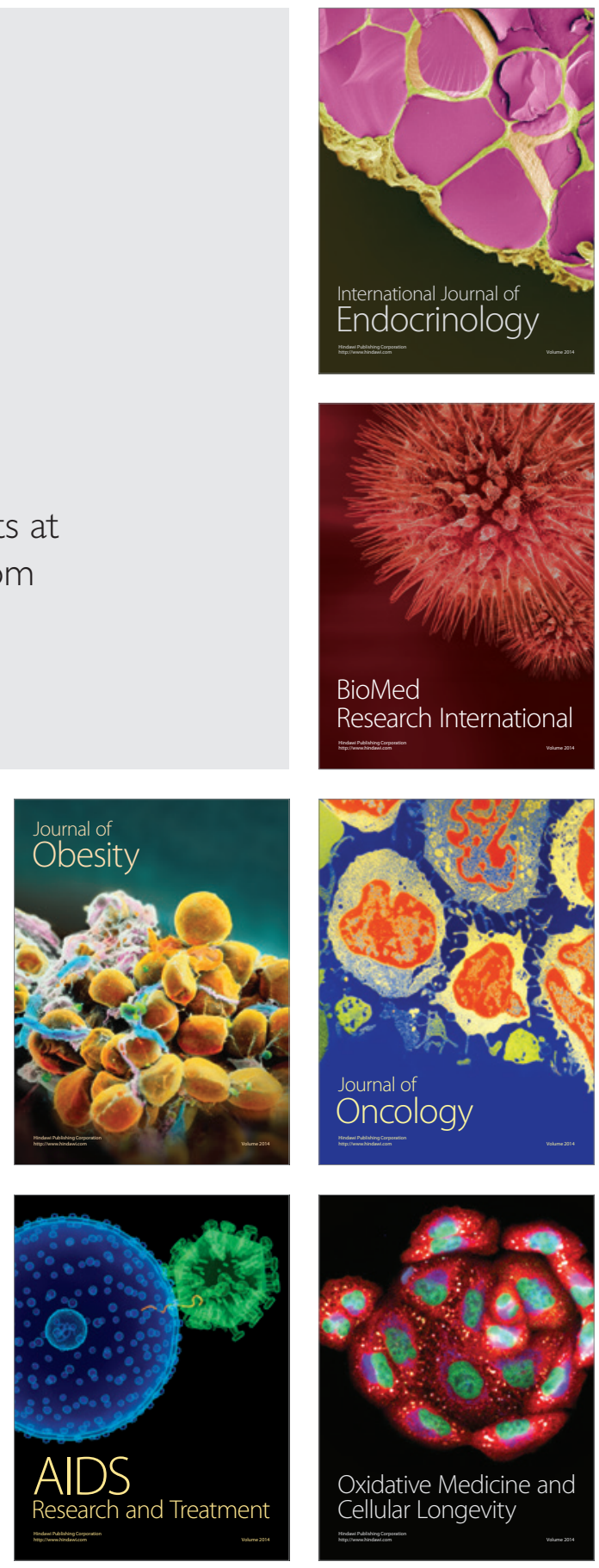